\title{
PSICOLOGIZACIÓN Y LENGUAJE EN EDUCACIÓN: ANALIZANDO DISCURSIVAMENTE POLÍTICAS EDUCATIVAS LATINOAMERICANAS CONTEMPORÁNEAS
}

\section{Psychologization and Language in Education: Discursively Analysing Contemporary Latin American Educational Policies}

Diego Palacios Díaz ${ }^{1}$

diegopalacios@uti.edu.ec

\section{Felipe Hidalgo Kawada ${ }^{2}$ \\ felipehidalgok@gmail.com}

\section{Paulina Saavedra Stuardo ${ }^{1}$}

psaavedra.stuardo@gmail.com
${ }^{1}$ Universidad Tecnológica Indoamérica, Ecuador

${ }^{2}$ Universidad de Sydney, Australia

Fecha recepción: 24/10/2019 Fecha aprobación: 26/11/2019

\section{Resumen}

El objetivo del presente artículo es analizar discursivamente cómo es incorporado el lenguaje psicológico en regulaciones contemporáneas de políticas educativas latinoamericanas, específicamente de Chile y Ecuador. La psicologización, tendencia a utilizar los saberes y técnicas de la Psicología y disciplinas afines para mirarnos a nosotros mismos, a los otros y al mundo social, representa una tecnología de cambio educativo que, paulatina y recurrentemente, se instala en diversos escenarios a nivel global. Conscientes de ello, y a la luz de los principios teóricos- metodológicos de los estudios críticos del discurso, se analiza un corpus de textos sobre política educativa de Chile y Ecuador, desde una perspectiva discursiva y social. Los principales hallazgos evidencian que los lenguajes psicológicos, articulados desde los dualismos constitutivos del discurso de la Psicología, contribuyen a la configuración del sujeto y el contexto de la intervención. Del mismo modo, los lenguajes psicológicos, configurados desde el ideal del equilibrio y la productividad, ofrecen una estrategia para abordar algunas problemáticas y metas educativas de los contextos analizados. Finalmente, estos hallazgos son discutidos y problematizados en función de los usos actuales del lenguaje psicológico y desde las posibilidades que contienen para articular posturas y prácticas educativas transformadoras.

Palabras clave: Chile, discurso, educación, Ecuador, Latinoamérica, políticas educativas, Psicología, psicologización.

\section{Abstract}

The objective of this article is to discursively analyze how psychological language is incorporated into contemporary regulations of Latin American education policies, specifically in Chile and Ecuador. Psychologization, a tendency to use the knowledge and techniques of Psychology and related disciplines to look at ourselves, at others and at the social world, represents a technology of educational change that gradually and recurrently settles in diverse scenarios at the global level. Aware of this, and positioned from the theoretical-methodological principles of critical discourse studies, we examine a set of texts on educational policy in Chile and Ecuador, analyzing them in a discursive and social perspective. The main findings show that psychological languages, articulated from the dualisms constituting the discourse of Psychology, contribute to the construction and shaping of the subject and the context of intervention. Similarly, psychological languages, configured from the ideal of balance and productivity, offer a strategy to generally address some educational issues and goals of the contexts analyzed. Finally, these findings are discussed and problematized based on current uses of psychological language and from the possibilities they contain to articulate transformative discourses and educational practices.

Keywords: Chile, Ecuador, education, educational policies, discourse, Psychology, psychologization.

ISSN (impreso): 2636-2139

ISSN (en línea): 2636-2147

Sitio web: https://revistas.isfodosu.edu.do/recie 


\section{Introducción}

La presente investigación analiza discursivamente cómo ha sido incorporado el lenguaje psicológico en regulaciones contemporáneas de políticas educativas latinoamericanas, particularmente de Chile y Ecuador. En un contexto de nuevas regulaciones globales en el campo de la educación, pretendemos examinar cómo los saberes y técnicas de la psicología y disciplinas afines han comenzado, paulatina y recurrentemente, a habitar los lenguajes de las políticas educativas, redefiniendo en vías creativas las formas en que comprendemos y abordamos los problemas que enfrentamos cotidianamente en las escuelas y en otros espacios de la vida social.

Nuestro punto de partida para estudiar las políticas educativas consiste en reconocer que ellas constituyen discursos, es decir, prácticas sociales que de diversas formas representan, imaginan y proyectan el mundo en que vivimos (Fairclough, 2003). Discursivamente, las políticas educativas crean circunstancias, marcos de acción y reglas del juego, donde los sujetos debemos desplegarnos para alcanzar determinadas metas que son, en ocasiones, compartidas, y en otras, impuestas. Sin embargo, su particularidad más relevante es que ofrecen orientaciones sobre quiénes somos o quiénes podríamos/deberíamos ser, y también sobre cuáles son los cursos de acción que podríamos/deberíamos seguir (Ball, 2013).

En tal escenario, esta investigación problematiza y analiza la trama discursiva de las regulaciones recientes de políticas educativas chilenas y ecuatorianas que, en las dinámicas de un mundo global, comparten un aspecto común: la utilización recurrente de los saberes y técnicas psicológicas para definir, orientar y, en algunos casos, modificar quiénes somos, cómo actuamos y cómo se estructuran las reglas del juego en educación. Este fenómeno, o más precisamente, esta tecnología de cambio educativo, ha sido denominada psicologización (De Vos, 2013), y refiere a las diversas y creativas formas en que utilizamos el lenguaje psicológico para mirarnos a nosotros mismos, a los otros y al mundo social.
Finalmente, en lo que respecta a la estructura del artículo, se presenta, en primer lugar, una perspectiva global y sociolingüística de las políticas educativas, además de los elementos que configuran nuestro enfoque metodológico. Posteriormente, analiza discursivamente textos de política educativa de Chile y Ecuador, para, finalmente, discutirlos en atención a cómo se despliegan los lenguajes psicológicos en las políticas educativas contemporáneas.

\section{Revisión de la literatura}

Se ha organizado la revisión de la literatura en dos secciones. La primera de ellas presenta una visión de contexto sobre algunas tendencias globales en el campo de las políticas educativas, para lo cual profundizamos en las racionalidades y tecnologías que orientan actualmente el cambio educativo. Posteriormente, caracterizamos las tecnologías de psicologización que han emergido en este campo, además de ofrecer una perspectiva sociolingüística para comprender su despliegue y operatoria en diferentes discursos de política educativa.

\subsection{Tendencias contemporáneas en política edu- cativa: una perspectiva global}

Actualmente, las políticas educativas son uno de los escenarios principales donde conviven, con relativa conflictividad, fuerzas nacionales y supranacionales (Fuller \& Stevenson, 2019). Cada vez más, los estados nacionales han desarrollado tendencias y trayectorias de políticas educativas que buscan responder y ajustarse a los lineamientos derivados de agentes supranacionales que organizan la gobernanza global, tales como el Banco Mundial (BM), el Fondo Monetario Internacional (FMI) y la Organización para la Cooperación y el Desarrollo Económico (OCDE), entre otras. Como ha sostenido Salhberg (2016), a modo de un germen (por el acrónimo GERM en inglés-Global Education Reform Movement), algunas racionalidades de política educativa viajan y se viralizan por el mundo, contagiando a sistemas educativos nacionales $y$, en la mayor parte de los casos, reestructurándolos en función de algunas orientaciones generales de política educativa 
que son funcionales a las lógicas de la globalización neoliberal (Ball, 2016).

Una de las racionalidades predominantes apunta a que los sistemas educativos afronten exigencias aparentemente contrapuestas: por una parte, los países deben articular procesos orientados a alcanzar los más altos estándares de calidad educativa; por otra, deben hacer esfuerzos conscientes por formar sujetos integrales que cuenten con un amplio equipamiento de capacidades y habilidades para enfrentar los desafíos de las complejas sociedades del siglo XXI. Esto ha conllevado al desarrollo de una competencia frenética entre naciones, regulada y arbitrada por la OCDE a través de las evaluaciones PISA (Programme for International Student Assessment) (Lewis, 2016; Rautalin et al., 2018) y, especialmente, a la instalación de diversas tecnologías de cambio educativo, ensamblajes de racionalidades prácticas y mecanismos que buscan, mediante metas más o menos conscientes, gobernar sujetos individuales y colectivos, grupos sociales y poblaciones (Rose, 1996, 1999).

En este nuevo escenario global de reforma educativa, las principales tecnologías de cambio educativo vigentes se orientan hacia la elección de las estrategias de privatización endógena y/o exógena como el medio más apropiado y eficiente para el aumento de la productividad y performance escolar (Ball, 2009), hacia la estandarización de currículos y procesos evaluativos como una estrategia de respuesta a las presiones derivadas de estados nacionales y agendas supranacionales (Verger et al., 2019), Todo ello orientado al desarrollo de estructuras de supravigilancia y accountability para la regulación y control de sujetos y su acción en las instituciones educativas (Falabella, 2019; Maroy et al., 2019), entre otras. De modo general, el despliegue de estas tecnologías ha sido funcional a los idearios y mecanismos que configuran la racionalidad neoliberal de Estado, particularmente en lo referido a su necesidad de reconfiguración y minimización de los estados nacionales (Harvey, 2015) y, especialmente, a su visión empresarial de mundo y a su concepción de sujeto como agente individual que actúa libre, racional y autónomamente en un escenario social atomizado (Ball, 2016; Foucault, 2006).

\subsection{Psicologización y lenguaje en educación: una perspectiva sociolingüística}

Aunque opera de forma más sutil que las tecnologías de privatización, estandarización y rendición de cuentas, la psicologización es un mecanismo que aparece recurrentemente en las políticas educativas contemporáneas, en lo que representa -siguiendo la metáfora de Salhberg (2016) - una mutación del germen. En términos generales, la psicologización refiere al uso de los vocabularios y esquemas explicativos de la psicología para mirarnos a nosotros mismos, a los otros y al mundo (De Vos, 2013), lo cual se puede relacionar con el intento sistemático de las élites y gobernantes de incorporar las disposiciones psicológicas de los ciudadanos como un objetivo central en el arte de gobernar (Rose, 1999). En dicho contexto, la psicologización es una tecnología de poder que se despliega a partir de las estrategias de gobernanza psicológica utilizadas por los estados nacionales, agencias supranacionales y agentes no estatales para gobernar a la población desde estos saberes y técnicas psicológicas (Pykett et al., 2017).

La psicologización en educación es un fenómeno de larga data, ya que la institucionalización disciplinar de la psicología se ha visto facilitada por los usos que las élites le han dado para abordar in situ y a distancia diversas preocupaciones de gestión y control de la población que confluyen en el contexto educativo (Moretti \& Energici, 2019; Parra, 2015). Actualmente, la principal novedad radica en que en las sociedades occidentales se promueve cada vez con mayor énfasis nuevas formas de autoridad terapéutica orientadas desde la necesidad de lograr un profundo conocimiento del mundo interior de los sujetos contemporáneos para ajustarlos a los objetivos de gobierno (Miller \& Rose, 2008). Una manifestación de ello es que la Psicología actual, metafóricamente, tiñe las agendas de política educativa, donde instala sus esquemas explicativos para abordar a los sujetos y contextos educativos desde las disposiciones psicológicas que tienen/carecen para afrontar los desafíos educativos (Palacios, 2018). De esta forma, cada vez son más frecuentes los esquemas de tipo problemasolución que evidencian, por ejemplo, que bajos ni- 
veles de rendimiento escolar están asociados, linealmente, con bajos niveles de autoestima y motivación académica, lo cual justifica, per se, la necesidad de diagnóstico e intervención psicológica a nivel individual y/o grupal en este ámbito.

Desde una perspectiva sociolingüística, la psicologización es una práctica discursiva (Foucault, 1992) que produce y disemina comprensiones folklóricas del lenguaje disciplinar de la Psicología y disciplinas afines en el ámbito de las políticas educativas. En tanto la trama discursiva de las políticas educativas se tiñe de psicologización, es preciso captar algunos movimientos específicos que configuran los lenguajes psicologizados con los que hoy abordamos la experiencia educativa. Uno de ellos tiene un carácter clásico y es posible situarlo en la filosofía de René Descartes: la organización dualista del lenguaje psicológico (Bekerman \& Zembylas, 2018). La emergencia del pensamiento cartesiano ha provisto a la Psicología de dos dualismos que organizan sus desarrollos teóricos. El primero de ellos es el dualismo MENTE/Cuerpo, el cual instala el supuesto de que existe un cuerpo material (res extensa) que es independiente de una mente inmaterial (res cogitans). Esto está conectado con el dualismo SELF/SOCIEDAD, el cual es de gran importancia para la Psicología y la educación, pues promueve la creencia de que existe un mundo interior (self) que absorbe y se nutre de un mundo exterior (sociedad), a pesar de que son mundos independientes.

Si examinamos la práctica educativa en las sociedades occidentales, es posible captar que el supuesto que guía la acción es que la actividad primaria del self, el pensamiento, se desarrolla más y mejor en condiciones de quietud y aislamiento, es decir, con independencia del mundo exterior. El self alcanza su plenitud únicamente cuando los objetos y situaciones del mundo exterior le son transferidas y enseñadas para que él logre construirlas internamente. Desde esta base, emergen otros dualismos que organizan nuestros modos de pensar y actuar en educación: NATURALEZA/CULTURA, COGNICIÓN/EMOCIÓN, BIENESTAR/MALESTAR, entre otros.
La psicologización como tecnología de cambio educativo y, de forma más específica, el lenguaje psicológico que ha colonizado el campo de las políticas educativas, se ha visto actualmente reconfigurado en torno a dos tendencias sociolingüísticas. La primera de ellas guarda relación con la emergencia de discursos catastróficos y apocalípticos en salud mental y carencias psicoemocionales que justifican necesariamente la intervención terapéutica en el campo educativo (Brunila, 2011; Ecclestone, 2016; Ecclestone \& Rawdin, 2016). Esto ha facilitado la emergencia, según Ecclestone (2017), de un nuevo dualismo: el de la AUTONOMía/vULNERABiLidAD, el cual orienta las racionalidades y estrategias de gobierno de la población desde el supuesto de que existen sujetos en situación de carencia (vulnerables) que deben ser intervenidos para ajustarse a los parámetros de la racionalidad neoliberal, vale decir, un sujeto racional, autónomo y que cumple sus propias metas (Brown, 2017; Brown et al., 2017). Esto genera que las escuelas sean consideradas como un sitio estratégico para intervenir psicológicamente a los sujetos, a la vez que estas intervenciones son consideradas cruciales para superar nudos críticos en políticas educativas y para modelar en diversas formas a los ciudadanos de las sociedades contemporáneas (Ecclestone \& Brunila, 2015).

La segunda tendencia sociolingüística es la neurologización de los problemas educativos. En un sentido general, esta tendencia describe el lugar que actualmente ocupan las neurociencias como un prisma que permite comprendernos a nosotros mismos, a los otros y al mundo. Según De Vos (2015), esto ha sido posible por las propias inconsistencias internas del lenguaje psicológico y el respaldo de cientificidad que ha encontrado la psicología en las neurociencias para justificar sus técnicas y procedimientos. Un ejemplo de ello es el considerable aumento de diagnósticos de Déficit Atencional con Hiperactividad en el contexto escolar, donde en la mayor parte de los casos se fundamenta en que existen dificultades neurológicas de base, y no problemáticas estructurales y socioculturales del contexto educativo, lo cual genera otro dualismo: CEREBRo/CONTEXTO. En definitiva, se viene promoviendo una visión somática de 
los sujetos (Rose, 2006), a la vez que los problemas educativos son inscritos en el ámbito de posibles dificultades estructurales y funcionales del cerebro, el nuevo centro de la conflictividad educativa (De Vos, 2016).

\section{Método}

Esta investigación se orienta, metodológicamente, desde los desde los Estudios Críticos de Discurso (ECD), un campo heterogéneo de teorías y métodos que se ocupan de estudiar el lugar y funcionamiento del lenguaje en las sociedades occidentales contemporáneas (Flowerdew \& Richardson, 2018). Los ECD son tributarios de diversas tradiciones teóricas de las Ciencias Sociales, dentro de las cuales destacamos los aportes de Michel Foucault y sus desarrollos en torno a la categoría discurso, la cual refiere a las prácticas sociales que sistemáticamente forman los objetos sobre los cuales hablan (Foucault, 1992), para quien, en toda sociedad, la producción, distribución y circulación del discurso están controladas y reguladas por un conjunto de procedimientos que posibilita (o no) hablar sobre distintos tópicos en ciertos márgenes espacio-temporales (por ejemplo, sobre los discursos que se promueven en las políticas educativas).

Respecto a lo anterior, la participación de los sujetos sociales en la producción, distribución y circulación de los discursos está mediatizada por las relaciones de poder (Foucault, 2008). Esto significa que las formas del decir están controladas por jerarquías sociales que regulan qué es lo posible de ser enunciado y qué no. Esto es una característica singular de las sociedades capitalistas contemporáneas, donde las relaciones de poder tienen una naturaleza parcialmente lingüística, puesto que el lenguaje es una de las vías privilegiadas para infundir determinadas visiones de mundo $y$, con ello, construir las hegemonías que gobiernan y modelan sociedades específicas (Fairclough, 2003).

Este asunto es especialmente relevante para el ámbito de la educación, donde las prácticas sociales suelen estar articuladas en torno a la dialéctica del amoesclavo. Como brillantemente planteó Paulo Freire
(2012), la práctica educativa está constituida por complejas cadenas de opresión que niegan la vocación histórica de los seres humanos de Ser Más. Esto se organiza mediante prácticas bancarias de naturaleza fundamentalmente narrativa, donde los opresores narran -o depositan- ciertas prescripciones que los oprimidos deben incorporar para aprender a narrar el mundo desde la perspectiva dominante. Siendo este un problema sociolingüístico, la apuesta de los ECD es justamente denunciar los aspectos lingüísticos que están en la base de las relaciones de poder y las jerarquías sociales (Fairclough, 2018), pero también constituyen un enfoque comprometido con anunciar nuevas condiciones de liberación y transformación para la vida humana (Palacios et al., 2019).

El campo de los ECD ofrece diversas herramientas y técnicas para analizar distintos tipos de discurso. Particularmente, en esta investigación, nos interesan las políticas educativas. Ellas se distinguen por algunos elementos que es necesario describir. En primer lugar, las políticas educativas están organizadas desde el razonamiento práctico (Fairclough \& Fairclough, 2012), es decir, formas de razonamiento que indican cuáles son los medios que deben utilizarse para alcanzar ciertos fines o, más específicamente, cuáles son las soluciones que podrían implementarse para abordar determinadas problemáticas. En segundo lugar, y a razón de lo anterior, las políticas educativas suelen mixturar tendencias indicativas y tendencias sugestivas. Esto implica que, en ocasiones, ellas indican abiertamente qué curso de acción debe seguirse, mientras que en otras más bien sugieren ciertas posibilidades (Ball, 2006). En tercer lugar, las políticas educativas nunca emergen ni actúan en un vacío institucional, sino que responden a determinadas circunstancias económicas, políticas, sociales y culturales, a partir de lo cual, configuran nuevos marcos de circunstancias para orientar la acción concreta de los sujetos e instituciones educativas.

\subsection{Textos y con-textos: procedimiento de Selec- ción Textual-Discursiva}

En esta investigación se analiza un conjunto de documentos sobre políticas educativas vigentes de Chile y 
Ecuador, examinando las tendencias de psicologización del lenguaje educativo. En esta sección quisiéramos situar brevemente estos textos, describiendo los contextos en los cuales emergen, los criterios por los cuales fueron seleccionados y la forma en que se abordaron dichos textos para analizarlos.

En la perspectiva global anteriormente explicada, Latinoamérica es un continente que presenta algunas particularidades estructurales. Fundamentalmente, esta región ha enfrentado por décadas las problemáticas de la pobreza, desigualdad socioeconómica y exclusión socioeducativa (Gentili, 2011). Desde una perspectiva de políticas educativas, esta región posee la mayor tasa de escuelas primarias privatizadas en el mundo (Verger et al., 2016), proceso que está acompañado de la participación de agencias supranacionales como el Fondo Monetario Internacional y la OCDE, quienes actúan como asesores en lo referente a la incorporación de tecnologías de privatización, estandarización y rendición de cuentas, y que además aportan, directa o indirectamente, a las reformas estructurales, incrementales o parciales de los estados nacionales de la región.

A razón de los distintos procesos macrohistóricos y las diferencias idiosincráticas de las distintas naciones latinoamericanas, las tendencias y trayectorias de privatización educativa han cobrado formas diferenciadas (Verger et al., 2017). Para lo que es de interés en nuestra investigación, es posible plantear que Chile y Ecuador representan los extremos opuestos de un continuo. Mientras Chile ha configurado, en las últimas décadas, un Estado mínimo a partir de un proceso de reforma estructural que ha privatizado de forma importante los derechos sociales elementales como la educación, Ecuador ha resistido fervientemente estas tendencias apegándose a una constitución plurinacional que garantiza el derecho a la educación en todas las etapas de la vida humana. Sin embargo, ambas naciones confluyen en la reciente incorporación de tecnologías de psicologización a sus programas de políticas educativas. Bajo el supuesto de que es necesario desarrollar una educación integral, las disposiciones actuales de política educativa de estos países aperturan novedosamente sus agendas para incorporar saberes y técnicas provenientes de las disciplinas psicológicas.

Con estos elementos en vista, escogimos algunas regulaciones de política educativa en torno a tres criterios: a) un criterio espacio-temporal que considerase aquellas regulaciones de Chile y Ecuador promulgadas en la última década (2010 en adelante); b) un criterio de pertinencia textual-discursiva que considerase aquellas regulaciones que, cumpliendo lo establecido en el primer criterio, evidenciara la incorporación de saberes y técnicas psicológicas como elemento central en sus formulaciones; c) un criterio de relevancia textual-discursiva que considerase aquellas regulaciones que, cumpliendo con lo establecido en los criterios anteriores, expresase de modo saliente racionalidades de política educativa fundamentadas en saberes y técnicas psicológicas.

A la luz de dichos criterios, se escogió, para el caso de Ecuador, la regulación Modelo de Funcionamiento de los Departamentos de Consejería Estudiantil (Ministerio de Educación de Ecuador, 2016) y la correspondiente Normativa para la implementación, organización y funcionamiento del Departamento de Consejería Estudiantil (Ministerio de Educación de Ecuador, 2016). En el caso de Chile, se escogió la normativa Otros Indicadores de Calidad Educativa (Ministerio de Educación de Chile, 2014) y el manual Programa Bienestar en la Escuela-Buen Clima Escolar (Ministerio de Educación de Chile, 2015). Los criterios utilizados y los textos escogidos se sintetizan a continuación en la Figura 1. De esta base textual, se escogieron algunos fragmentos de interés que fueron analizados con los procedimientos que detallaremos a continuación para cumplir con los propósitos de esta investigación. 


\section{Figura 1. Criterios de selección textual-discursiva y textos escogidos}

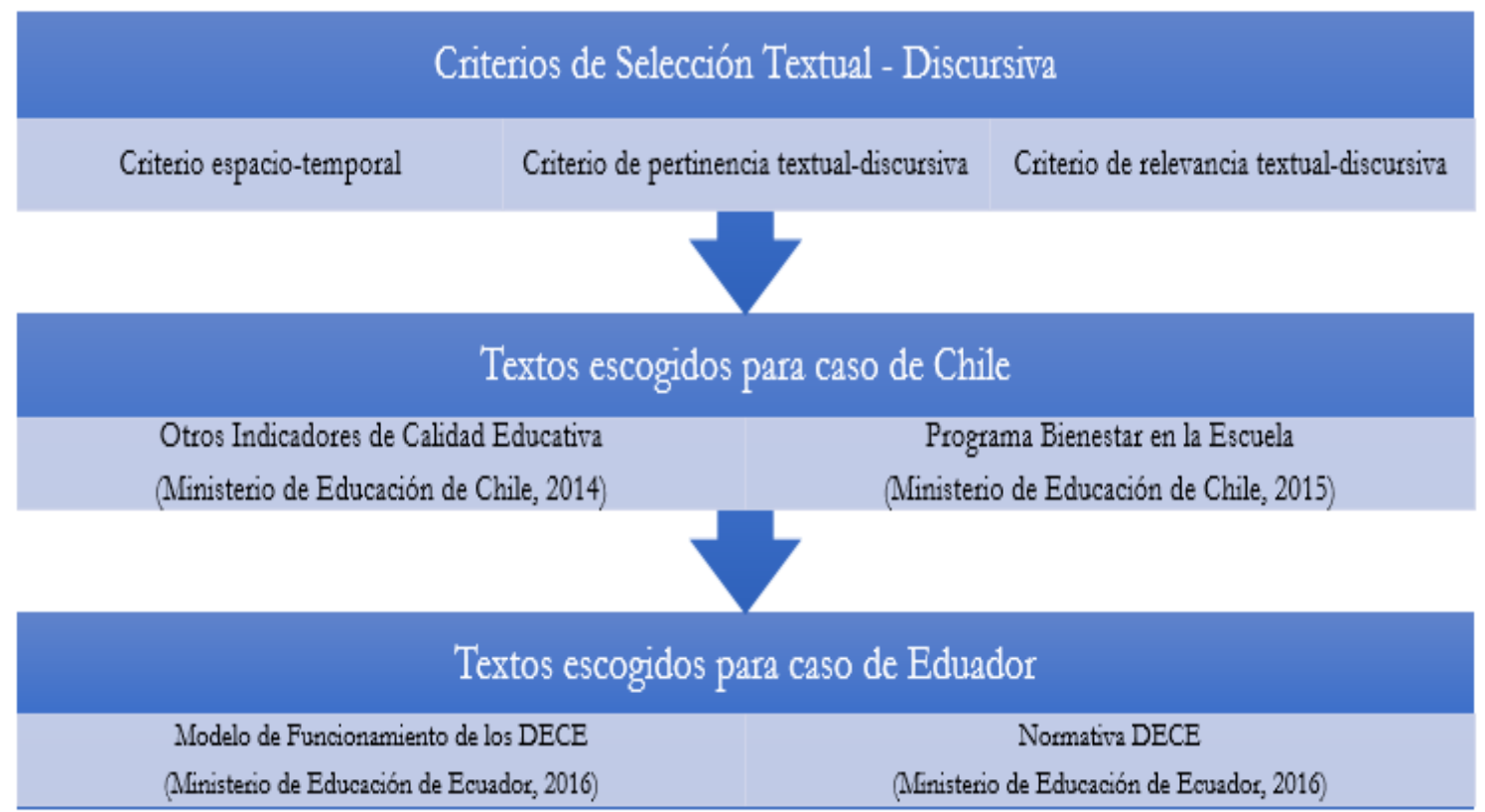

Nota: Elaboración propia.

\subsection{Abriendo la caja de herramientas: ruta y es- trategias analíticas}

Trazamos una ruta analítica que contempla dos niveles: un nivel discursivo y un nivel social (Figura 2). De esta manera, una tarea primaria fue la elaboración de una caja de herramientas de análisis discursivo configurada desde distintos enfoques pertenecientes al campo de los ECD. Consideramos primeramente el ACD textualmente orientado de Norman Fairclough (2003). Este enfoque aborda los aspectos funcionales del lenguaje, es decir, qué es lo que los discursos en tanto prácticas hacen [efectos] en distintos niveles de la realidad social. Concretamente, dentro de las diversas posibilidades que ofrece este modelo, escogimos como herramienta principal las imbricaciones de géneros (modos de hacer), discursos (modos de representar/proyectar) y estilos (modos de ser) que subyacen en toda práctica discursiva y social, a fin de examinar los efectos promovidos por las distintas regulaciones contempladas.

Secundariamente, consideramos el Análisis Político de Discurso (APD) de Fairclough y Fairclough (2012). Este enfoque atiende a la especificidad del discurso político, pesquisando las distintas conexiones de medios- fines que se ofrecen en las argumentaciones, así como también las circunstancias en que emergen y los valores que guían la elección de determinados medios. Concretamente, utilizamos como herramientas el análisis de las conexiones medios- fines y las definiciones de circunstancias en que emergen determinadas soluciones de política educativa en las distintas regulaciones analizadas.

Para el nivel social, formulamos algunas preguntas generales que orientaron nuestro análisis tras examinar discursivamente los textos. Concretamente, nos interrogamos: ¿de qué formas son incorporados los saberes y técnicas de la disciplina psicológica en las políticas educativas?, ¿qué problemas se orientan a solucionar el saber y lenguaje psicológico?, ¿a través de qué medios, estrategias y tácticas?, ¿cómo son caracterizadas las escuelas y distintos sujetos educativos en los lenguajes psicológicos?, ¿qué convergencias/ divergencias existen entre los escenarios?, ¿qué posibilidades de cambio y alternativa pueden establecerse ante la dominancia de los lenguajes psicológicos en la política educativa? 


\section{Figura 2. Niveles de análisis}

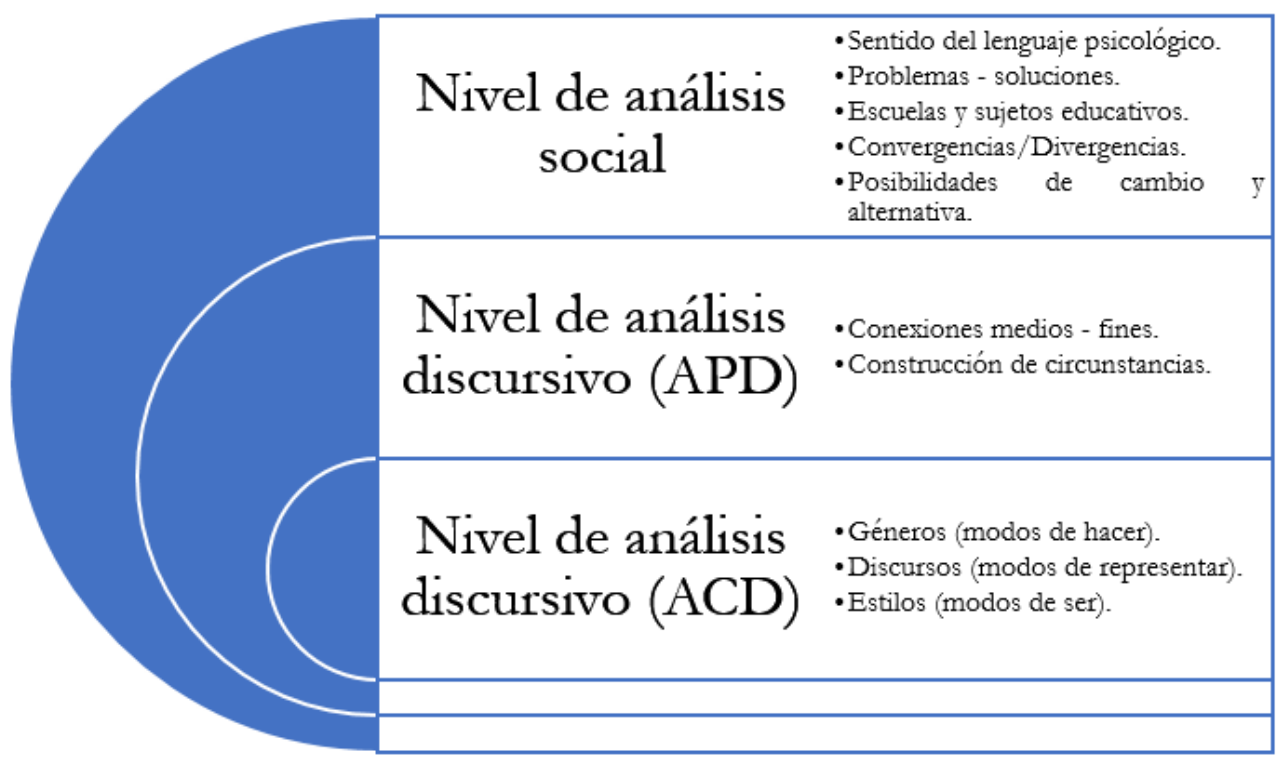

Nota: Elaboración propia.

\section{Resultados}

Se ha organizado la presentación de los resultados a partir de un análisis diferenciado de cada uno de los contextos, atendiendo a las singulares formas en que los lenguajes psicológicos emergen y son utilizados en las políticas educativas. Aun cuando se evitó relevar la particularidad de cada escenario, en algunos momentos hemos establecido conexiones a partir de sus similitudes, las cuales serán abordadas con mayor detalle en las discusiones y conclusiones de este artículo.

\subsection{Chile: ampliando la calidad educativa}

El sistema educativo chileno ha generado una novedosa estrategia de gobierno basada en los aportes provenientes de los saberes y técnicas psicológicas. En 2014, el Ministerio de Educación, en conjunto con una comisión de expertos e investigadores del ámbito de la Psicología y educación, seleccionaron y elaboraron un conjunto de indicadores psicosociales orientados a ampliar la noción de calidad educativa. Tradicionalmente circunscrita al rendimiento de los estudiantes en la prueba estandarizada Simce (Sistema de Medición de la Calidad Educativa) la noción de calidad fue ampliada a partir de la integración de otros elementos necesarios de atender en las escuelas: autoestima académica y motivación escolar, clima de convivencia escolar, participación y formación ciudadana y hábitos de vida saludable. Ellos, en conjunto con algunos indicadores sociotécnicos más específicos, configuran los Otros Indicadores de Calidad (OIC)

un conjunto de índices que entregan información sobre aspectos con el desarrollo personal y social de los estudiantes, en forma complementaria a la información proporcionada por los resultados Simce y los Estándares de Aprendizaje, ampliando de este modo la concepción de calidad educativa (Ministerio de Educación de Chile, 2014, p.5).

En un sentido estratégico, los OIC aportan información complementaria a la que ya proveen otros mecanismos de evaluación educativa en Chile. Esta información cumple dos fines en el marco de las regulaciones vigentes de política educativa, ya que, en principio, ordena a los establecimientos educativos en función de categorías de desempeño, lo cual sir- 
ve, a su vez, para informar a las familias sobre cuáles son los niveles de calidad educativa de los establecimientos educativos que deben escoger para sus hijos. Los mecanismos de ordenamiento e información perfecta para orientar la toma racional de decisiones son constitutivos del cuasi mercado educativo que se despliega en Chile hace cuatro décadas. Los OIC se insertan en este conjunto de discursos y prácticas pro-mercado educativo para redefinir en vías creativas las disposiciones psicológicas de estudiantes y, con ello, reorganizar la vida escolar. Concretamente, conviene examinar el caso de dos indicadores psicológicos: autoestima académica y motivación escolar. La regulación de los OIC indica que

La autoestima académica y la motivación escolar son dimensiones íntimamente relacionadas y que se influencian mutuamente. Un estudiante que se siente capaz académicamente es más probable que se interesa e invierta esfuerzo en las actividades escolares. Asimismo, un estudiante que está motivado e interesado por el estudio y el aprendizaje probablemente se esfuerce más y obtenga resultados que beneficien su autoestima académica. Es decir, la autoestima y la motivación académica influencian conjuntamente la forma en que los estudiantes piensan, sienten y se comportan en relación con las actividades escolares (Ministerio de Educación, 2014, p.17).

Existen algunos elementos de interés analítico en el fragmento expuesto. En primer lugar, las argumentaciones de política educativa son articuladas en términos de influencias lineales y probabilidades, una tendencia propia del lenguaje psicológico enmarcado en el paradigma positivista. Por ejemplo, un nivel alto de autoestima podría permitir [es más probable] mayor interés y esfuerzo en la vida escolar. De la misma manera, un alto nivel de motivación escolar contribuiría [probablemente] a esforzarse más y obtener mejores resultados. De ello se desprende, en segundo lugar, la proyección de un homo psychologicus-scolae: un sujeto que desde sus disposiciones psicológicas aumenta sus probabilidades de obtener mejores resultados escolares. La autoestima escolar y motivación académica quedan definidas como medios de aumento del esfuerzo y del logro escolar, y a la vez como un intento de conjuro de los azares que se movilizan en la dicotomía éxito/fracaso escolar. En tercer lugar, y de forma explícita, se evidencia una racionalidad instrumental que promueve que estos indicadores psicosociales influencian conjuntamente [medio] la forma en que los estudiantes piensan, sienten y se comportan en relación con las actividades escolares [fin].

El blanco del cálculo y la intervención de gobierno a través de estos lenguajes psicologizados es el homo psychologicus-scolae, un sujeto que es comprendido e intervenido desde las disposiciones psicológicas que aumentan su productividad escolar. Es interesante notar cómo esta forma de incorporar el lenguaje psicológico fortalece la dicotomía SELF/SOCIEDAD, donde el mundo interno de los sujetos aparece desconectado de los aspectos que constituyen el mundo externo. La siguiente forma de abordar el fenómeno del aprendizaje sitúa la actividad en el mundo interno del sujeto, restando importancia a las propiedades que caracterizan el mundo externo:

Aprender requiere un involucramiento activo por parte de los estudiantes: es necesario poner atención, dedicar tiempo y esfuerzo, y posponer otras actividades. Nada de esto ocurre si perciben que lo que deben aprender no es estimulante y les aporta poco a sus vidas [...] el 91\% de los estudiantes chilenos piensan que el colegio ha sido una pérdida de tiempo y la mayoría declara que no le gusta leer ni disfruta de la Matemática (Ministerio de Educación, 2019, p.19).

El efecto que se promueve discursivamente en esta regulación, como hemos planteado, apunta a perfeccionar aquellas disposiciones psicológicas que aumentan la productividad escolar. El dato referido a la apreciación de los estudiantes chilenos sobre la experiencia escolar deja entrever que la sensación de pérdida de tiempo y de disgusto por las materias escolares, es una problemática interna, que no necesariamente está relacionada con las particularidades que organi- 
zan la experiencia escolar. El homo psychologicus-scolae debe encontrar, en las profundidades del self, el estímulo y el aporte específico que la Educación Escolar aportará a sus vidas. Es responsable primario y activo de su progreso: necesita poner atención, necesita dedicar tiempo y esfuerzo, necesita posponer otras actividades. Su vida está circunscrita a una escuela estéril de responsabilidades, pues pase lo que pase en ella, lo valioso y valorado es la actividad emprendida por el propio sujeto.

El lenguaje psicológico tiñe de formas creativas los modos de organización de la vida escolar. La regulación de los OIC entrega, con tonalidad sugestiva que transita hacia lo indicativo, un conjunto de recomendaciones sobre qué es lo que hacen aquellas escuelas exitosas en la gestión de los indicadores psicosociales que se encuentran en la base de una noción ampliada de calidad educativa. Por ejemplo, dentro de un amplio set de medidas, esta regulación indica que aquellas escuelas que promueven el desarrollo de la autoestima académica y la motivación escolar entre sus estudiantes

\section{Muestran a los estudiantes que ellos pueden emprender acciones para superar sus pro- blemas.}

Los profesores muestran a los alumnos que los fracasos dependen de factores que ellos pueden cambiar (por ejemplo, técnicas de estudio, constancia, tiempo dedicado) y no de causas fuera de su control (por ejemplo, falta de habilidades), que los errores son oportunidades para aprender y que sus progresos se deben al esfuerzo que cada uno ha invertido (Ministerio de Educación de Chile, 2014, p.22).

Esta sugerencia representa una actualización del dualismo SELF/SOCIEDAD, presente en la estructuración del lenguaje psicológico que caracteriza a esta regulación, aunque es también una incorporación novedosa de aquellas corrientes psicológicas orientadas hacia el éxito personal y empresarial (coaching, PNL, entre otros). Un sujeto del mundo externo, como el profesor, es responsable de mediar en el mundo interno de los estudiantes. Los problemas se despliegan en su mundo interno, mientras el contexto en el que se desenvuelven se mantiene estéril y aséptico. El estudiante, organizado desde los esfuerzos que realiza para aprender, debe estar enfocado en los factores que puede controlar y en convertir los errores en oportunidades. Aislado del mundo en que vive y ausente de las relaciones sociales, el homo psychologicus-scolae se regocija individualmente de sus logros porque son expresión de una ecuación infalible: más esfuerzo individual, más éxito individual (probablemente).

Las orientaciones promovidas en los OIC encuentran respaldo en otros documentos ministeriales que integran saberes y técnicas psicológicas para abordar las problemáticas educativas. En el manual Programa Bienestar en la Escuela (Ministerio de Educación de Chile, 2015), se ofrece "un apoyo al trabajo de muchos docentes y otros adultos que se ven sometidos diariamente a exigencias y demandas que, a menudo, constituyen factores de creciente estrés y ansiedad" (p.4). El propósito principal de este documento es mejorar el clima emocional en las escuelas mediante la promoción de estados de mayor bienestar y armonía. Es novedosa la incorporación realizada en esta regulación en términos de los fundamentos teóricos del bienestar

La doctora Amanda Céspedes, destacada neuropsiquiatra y pionera en el estudio y práctica de esta ciencia en nuestro país, define Bienestar como "un estado de armonia que se produce del equilibrio entre las demandas que nos llegan del mundo exterior y los recursos emocionales que tenemos para enfrentarlas". A su vez, Ignacio Morgado, reconocido psicobiólogo español, expresa "resulta imposible separar el bienestar del estado emocional de las personas". Esta aseveración cobra una especial relevancia en nuestro contexto sociocultural de claro predomino de la razón [...] La gran interrogante es cómo potenciamos estos estados de armonía que permitan ampliar el clima de bienestar en nuestras vidas, disminuyendo la intensidad del malestar. Y cómo, a su vez, estos hallazgos pueden convertirse en instrumentos pedagógicos para ser utilizados en las escuelas (Ministerio de Educación de Chile, 2015, p.5). 
La emergencia del dualismo RAZÓN/EMOCIÓN moviliza las argumentaciones que se promueven en este documento. El contexto cultural, caracterizado de forma predominantemente racional, obstaculiza las posibilidades de potenciar el bienestar (emocional-implícito) y disminuir el malestar (emocional-implícito). La necesidad de desarrollar instrumentos pedagógicos para la vida escolar que cumplan dicho fin se fundamenta en dos disciplinas de alta vigencia por la funcionalidad que tienen sus supuestos para las perspectivas psicológicas dominantes en educación: la Neuropsicología y la Psicobiología. Una confluencia que observamos en ambas disciplinas es que promueven una actualización del dualismo SELF/SOCIEDAD, a partir del supuesto de que existe un mundo exterior que desafía y exige al mundo interno y que genera alteraciones del ideal estado de equilibrio que debe organizarlo. El supuesto del equilibrio es, a su vez, lo que organiza las acciones de cálculo e intervención psico-neurológicas en el contexto escolar que promueve este documento. Así lo evidencia una viñeta propuesta para ritualizar el inicio de la jornada escolar

\section{Figura 3. Recomendaciones para el inicio del día}

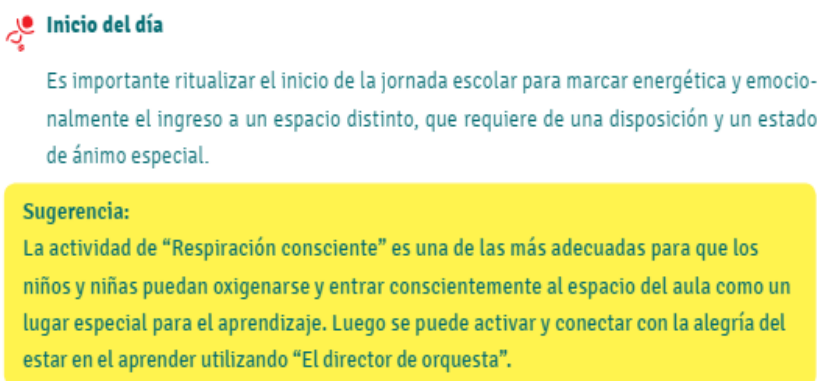

Nota: Ministerio de Educación de Chile (2015, p.12).

En este registro, las disposiciones psicológicas son abordadas en un nivel psicobiológico y neuropsicológico. El self precisa de un estado energético y emocional apropiado (mundo interno) para ingresar a un espacio distinto (mundo externo). Desde el supuesto que para entrar a la sala de clases se precisa de una disposición y un estado de ánimo especial, las disposiciones internas son trabajadas en pos de lograr el equilibrio, para la cual el profesor debe utilizar una "voz calmada e instrucciones claras y pausadas y así evitar distracciones, hiperventilación o desinterés" (p.6), en el caso de la actividad Respiración Consciente, y "estimular la concentración y la atención a través de la escucha musical" (p.50) en la actividad Director de Orquesta.

Es importante notar que, independientemente del fundamento teórico y la visión educativa defendida en las psico-neuro-disciplinas, lo que es relevante para la práctica educativa son los mecanismos que participan en la construcción de las disposiciones que precisan los sujetos educativos contemporáneos para lograr determinados fines. En este escenario, se promueve explícitamente la noción de un sujeto que está internamente equilibrado, en el plano energético y emocional, afrontando los desafíos y exigencias de un mundo externo que opera como mero escenario de desenvolvimiento. Implícitamente, los efectos promovidos son un sujeto aislado, posiblemente exonerado de las relaciones sociales, que se dispone heroicamente a afrontar las adversidades, ideario que conecta con las hegemónicas visiones liberales en educación y en otros ámbitos de la vida social. Una muestra de ello son las recomendaciones para enfrentar la evaluación que se derivan de estas disciplinas:

\section{Figura 4. Recomendaciones para enfrentar una prueba de alta exigencia}

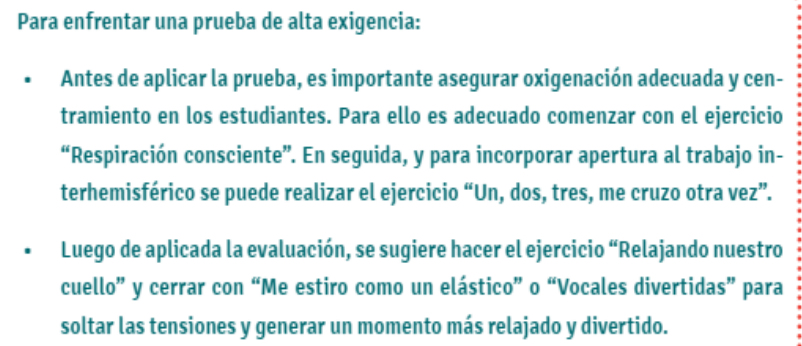

Nota: Ministerio de Educación de Chile (2015, p.15).

La ritualización de las prácticas educativas es, en definitiva, el aporte distintivo que ofrecen las disciplinas psicológicas a las agendas contemporáneas de políticas educativas en Chile. Es interesante apreciar un doble movimiento en esta figura. Lo primero es 
que no se cuestiona la alta exigencia, pues esta constituye un fin necesario. Lo que sí es posible de alcanzar es un mayor ajuste del sujeto (mundo interno) a las condiciones que le exigen y desafían (mundo externo), pero no al revés. El segundo movimiento consiste en abordar e intervenir los fundamentos más íntimos del sujeto que aprende y que es evaluado, situados hoy a nivel interhemisférico, logrando que, para rendir adecuadamente, se oxigene adecuadamente y se centre. Estas visiones asépticas de la educación apelan en favor de eliminar cualquier tipo de disrupción, y desechando, por lo tanto, las situaciones de desequilibro y conflicto como potenciadoras de aprendizajes. En este sentido, la oxigenación y centramiento constituyen, de algún modo, técnicas de control en favor de la productividad escolar como fin en sí mismo. Posteriormente a la evaluación, y como forma lúdica de matizar el control, se proponen actividades orientadas a soltar tensiones y propiciar un momento más relajado y divertido, lo cual homologa evaluación con tensión, aspecto característico de las prácticas educativas actuales y que, posiblemente, expliquen la crisis de sentido y pertinencia que tiene la Educación Escolar hoy que, como ellos mismos seńalan, alcanza al 91\% de los estudiantes chilenos.

\subsection{Ecuador: transición y equilibrio}

En 2016, y como parte de las estrategias gubernamentales orientadas hacia el desarrollo de la filosofía del Sumak Kawsay (Buen Vivir-Vida en Armonía), Ecuador incorporó en sus regulaciones de Política Educativa a los Departamentos de Consejería Estudiantil (DECE).

Un organismo dentro de las instituciones educativas que apoya y acompaña la actividad educativa mediante la promoción de habilidades para la vida y la prevención de las problemáticas sociales, fomenta la convivencia armónica entre los actores de la comunidad educativa y promueve el desarrollo humano integral bajo los principios de la Ley Orgánica de Educación Intercultural. A su vez, busca que la experiencia educativa trascienda la adquisición y acumulación de conocimientos a la cons- trucción del propio conocimiento enfocado en principios, valores y herramientas reflexivas para el desarrollo personal, la autonomía, la participación ciudadana y la construcción de proyectos en el marco del Buen Vivir (Ministerio de Educación de Ecuador, 2016, p.12).

Estratégicamente, los DECE son una regulación creada para abordar, desde enfoques preventivos y promocionales, distintas problemáticas psicosociales y sociales que confluyen en las escuelas ecuatorianas. Esta regulación incorpora, según el tamaño de la matrícula de cada institución y sus respectivas necesidades psicosociales, un equipo de psicólogos y trabajadores sociales cuya misión es fomentar la convivencia armónica entre distintos actores y, en un sentido amplio, contribuir a alcanzar las aspiraciones de gobierno de la población. Es interesante, desde nuestro foco analítico, la transición que se presenta desde una experiencia educativa centrada en la adquisición y acumulación de conocimientos hacia el desarrollo de un conocimiento propio que posee aspectos no necesariamente académicos. La particularidad de este conocimiento es que promueve una doble perspectiva compuesta por lo individual (desarrollo personal-autonomía) y lo social (participación ciudadana-construcción de proyectos), lo cual, implícitamente, posiciona la dimensión formativa del ser humano por sobre [trasciende] la dimensión académica.

En correspondencia con lo anterior, esta regulación se articula desde diversos enfoques teóricos que brindan líneas de acción para los profesionales: intercultural, bienestar, pedagógico e inclusivo, entre otros. Particularmente, ellos posibilitan el ingreso y, en algunos casos, la actualización de algunas perspectivas psicológicas que subyacen a las prácticas educativas. $\mathrm{Al}$ respecto, es interesante la forma en que el enfoque de desarrollo humano actualiza el dualismo MENTE/ CUERPo en torno al objetivo del equilibrio, el cual aparece matizado en el verdadero encuentro con sí mismo, los otros y la naturaleza, pero también en torno al cultivo de la reflexión personal y la acción orientada por determinados valores. 
El enfoque de desarrollo humano busca encontrar el equilibrio entre el cuerpo y la mente, vivir un verdadero encuentro con uno mismo, con los demás y con la naturaleza mediante la reflexión personal en torno a las diferentes formas de actuar desde el cuidado de sí, el respecto incondicional, la coherencia y la empatía (Ministerio de Educación de Ecuador, 2016, p.12).
La noción de homo psychologicus-scolae que ofrecimos para el caso de Chile emerge con algunos matices en las regulaciones de política educativa ecuatoriana. En este caso, el ser humano es concebido de forma tridimensional, donde al tradicional dualismo SELF/soCIEDAD (dimensión personal y dimensión sociocultural) se suma una dimensión espiritual que actualiza de forma novedosa el dualismo MENTE/CUERPO, tal como se aprecia en la Figura 5.

\section{Figura 5. Dimensiones del Ser Humano}

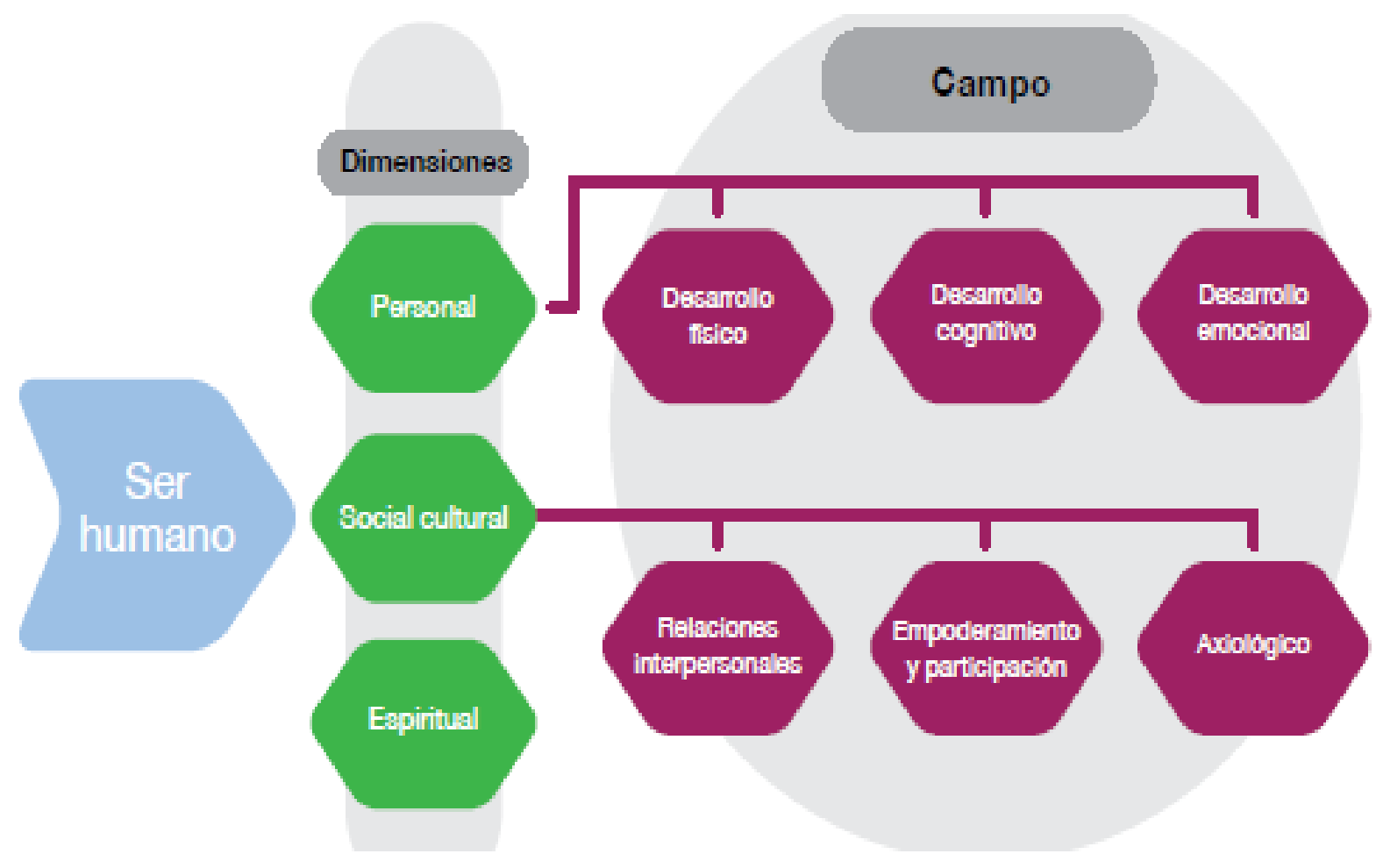

Nota: Ministerio de Educación de Ecuador (2016, p.6).

En esta regulación, la dimensión personal del ser humano está "conformada por el desarrollo físico, cognitivo y emocional, aspectos que están íntimamente relacionados y que construyen la identidad personal" (Ministerio de Educación de Ecuador, 2016, p.6). Esta integración de elementos que aparecen cualitativamente diferenciados (cuerpo-pensamiento-emociones) se relaciona con la dimensión sociocultural, donde se comprende que "la cultura y la sociedad constituyen elementos externos que forman al ser humano mediante pautas culturales que influyen en la conformación de la personalidad, las conductas, el pensamiento y la relación con el medio" (p.6). Si analizamos este asunto desde el dualismo $S E L F /$ soCIEDAD, no sólo apreciamos nuevamente cómo este ocupa un lugar central en los discursos psicológicos que tiñen la política educativa, sino que advertimos cómo se promueve y refuerza la idea de que el self (interno) incorpora pasivamente aspectos socioculturales (externo), el cual entrega activamente pautas 
culturales que van moldeando el mundo interior. De hecho, la dimensión espiritual planteada en esta regulación contribuye, discursivamente, a armonizar lo que, eventualmente, representa una oposición de fuerzas (ser humano y el mundo que nos rodea). Esta dimensión se comprende como

aquella que trasciende el mundo de lo físico y que permite vincularnos con todos los seres vivos en armonía, mediante principios que orientan la interrelación de la persona, la sociedad y la naturaleza. A esta visión espiritual, es necesario abonarla con la filosofía del Sumak Kawsay (Vida en Armonía), perspectiva que nos permite comprender la necesidad de potenciar una relación equilibrada desde el ser humano con el mundo que nos rodea (Ministerio de Educación de Ecuador, 2016, p.7).

Esta regulación y, particularmente, esta concepción de ser humano, es una ruta general para orientar el quehacer de los profesionales DECE. El homo psychologicus-scholae bosquejado en las regulaciones chilenas, adquiere aquí la forma de un homo psychologicus-vulnerable, un sujeto que requiere de múltiples intervenciones para conjurar los peligros y riesgos de una sociedad que "presenta nuevos e inquietantes desafíos en la tarea de formar y orientar para la vida” (Ministerio de Educación de Ecuador, p.5). Ciertamente, los saberes psicológicos se encuentran en la base de estas formas de abordar la conflictividad social contemporánea. Por ejemplo, el enfoque de bienestar que guía el presente modelo,

permite reconocer la necesidad de desarrollar estrategias enmarcadas en la comprensión de los diferentes procesos psicológicos (afectivos, emocionales y subjetivos) que acompañan el desarrollo de niños, niñas y adolescentes, la inclinación y escucha activa de la situación personal del estudiante, su contención psicológica-emocional en casos de vulneración de derechos [...] (Ministerio de Educación de Ecuador, 2016, p.7).

Esta particular forma de integrar el lenguaje psicológico a las políticas educativas perfila al estudiante desde los procesos psicológicos, fundamentalmente emocionales, que acompañan de forma estable su desarrollo, pero que también están presentes en situaciones que transgreden sus derechos fundamentales. Metafóricamente, la apelación a la acción de "contención”, promueve el efecto de que las emociones son una fuerza que, en ocasiones, desborda ciertos límites. Desde dicho supuesto, el trabajo de los profesionales DECE se orienta a bosquejar dichos límites y a atender a situaciones específicas a fin de mantener el equilibrio del sujeto ante un contexto que presenta recurrentemente "situaciones de riesgo como son los distintos tipos de violencia y maltrato (físico, sexual, verbal, emocional), la tenencia y consumo de drogas, embarazos en adolescentes, inadecuada utilización de las tecnologías de la comunicación e información, entre otras problemáticas sociales" (Ministerio de Educación de Ecuador, 2016, p.9).

El resguardo del equilibrio del homo psychologicus-vulnerable y su contención en situaciones de riesgo, se orienta desde algunos principios que los profesionales DECE deben cumplir. Nuevamente, es interesante notar cómo los lenguajes psicológicos prescriben y preforman ciertas directrices de acción para los sujetos, dentro de las cuales destacan:

- La asertividad como una expresión consciente a través de la cual el profesional comunica las ideas desde un estado interior de autoconfianza en donde no agrede ni se somete a la voluntad de otras personas, sino que manifiesta sus convicciones y defiende sus ideas de forma consciente, congruente, directa y equilibrada.

- La confidencialidad como una práctica constante que invita a trabajar con ética profesional, generando relaciones profesionales de confianza y seguridad basadas en el respeto y la reserva de información recibida.

- La empatía como la habilidad para percibir al otro en sus sentimientos y emociones, colocándose en la realidad de la otra persona, siendo capaz de reconocerse e identificarse con los demás (sentir con el otro) (Ministerio de Educación de Ecuador, 2016, p.12). 
Finalmente, nos parece relevante indicar cómo esta incorporación del lenguaje psicológico, además de ofrecer una directriz técnica para las líneas de acción que deben seguir los profesionales, presenta también una regulación moral que, articulada desde el ideal del equilibrio y la reflexividad, orienta las formas en que dichos sujetos deben comportarse para el resguardo y contención de los estudiantes. Lo emocional y lo moral se entrecruzan para definir principios orientados a salvaguardar aquellas situaciones que, real o potencialmente, rompan el equilibrio y la armonía que incesantemente aparecen en las aspiraciones educativas de Ecuador.

\section{Discusión y conclusiones}

A modo de conclusión, hemos observado cómo la incorporación y uso del lenguaje psicológico en las regulaciones recientes de política educativa chilena y ecuatoriana constituyen una estrategia para abordar aspectos problemáticos de la vida social y educativa de estos países, en donde la tendencia ha sido la incorporación de estos lenguajes para generar nociones ampliadas o más trascendentes de calidad educativa, uno de los principios que orienta los esfuerzos educativos en Latinoamérica. Preferentemente, a través del indicador discursivo de la integralidad (educación integral, formación integral), se desarrollan estrategias orientadas a superar el énfasis exclusivo de la vida escolar en materias tradicionales, las cuales propenden a generar indicadores complementarios, como observamos para el caso de Chile, o a incorporar apoyos psicológicos para trascender hacia el conocimiento de lo propio, como ocurre en el caso de Ecuador.

Esta preocupación por la integralidad aborda preocupaciones y problemáticas específicas de política educativa desde distintas referencias. En el caso de Chile, se aprecia una creciente preocupación por la productividad escolar, organizada desde el supuesto de que, interviniendo al sujeto en sus disposiciones psicológicas, existirá una tendencia a aumentar en rendimiento y performance educativa. A su vez, hay que mencionar que las políticas de la última década, paralelamente a esta construcción de sujeto deseable de rendir exitosamente en las pruebas censales nacio- nales e internacionales, han pretendido colocar a la institución escolar como un espacio privilegiado para que los jóvenes puedan adquirir cuestiones relacionadas con su salud. En medio de los procesos de medicalización que viven las sociedades contemporáneas, procesos a los cuales la escuela no ha estado ajena, la búsqueda por el bienestar, los hábitos de vida saludable, el equilibrio psicoemocional, entre otros indicadores, se constituyen en aquellos indicadores que podrían atenuar o frenar las altas cifras de sedentarismo, obesidad infantil y trastornos ansioso-depresivos que se vienen constatando en la población escolar y adulta de Chile.

Los lenguajes psicológicos incorporados en las políticas educativas chilenas configuran al sujeto en tanto homo psychologicus-scolae, a la vez que proponen un amplio contexto de posibilidades de mejora/intervención que apuestan por un aumento cuantitativo en las disposiciones psicológicas para aumentar rendimiento escolar, así como también a una novedosa neurologización (De Vos, 2015), de algunas de las intervenciones que buscan ritualizar la vida cotidiana en la escuela desde el supuesto de que se requiere un estado orgánico equilibrado para participar de la experiencia educativa.

En las políticas educativas ecuatorianas se presenta discursivamente un contexto apocalíptico (Ecclestone, 2016; Ecclestone \& Rawdin, 2016), donde en la escuela y en la sociedad confluyen diversos riesgos que alteran el equilibrio y el sano desarrollo de niños y adolescentes, lo cual justifica el despliegue de las intervenciones preventivas y promocionales que son responsabilidad de los DECE. El homo psychologicus-vulnerable es el blanco de dichas intervenciones, lo cual constituye una forma de pensar el lugar del sujeto y, particularmente del estudiante, desde las carencias de distinta índole que lo constituyen y desde reales o potenciales riesgos a los que se ve enfrentado en su trayectoria escolar (Ecclestone, 2017).

Se observan algunas confluencias discursivas entre los contextos que son necesarias de destacar por las tendencias que marcan en las políticas educativas examinadas. Los lenguajes psicológicos utilizados es- 
tán articulados desde los clásicos dualismos que organizan el discurso disciplinar de la Psicología, y son actualizados en función de ciertas aspiraciones estratégicas de cada uno de los contextos. Desde nuestra óptica, es necesario atender y problematizar la forma en que el dualismo SELF/SOCIEDAD es incorporado en ambos contextos. Este dualismo promueve consistentemente que existe un mundo externo (sociedad) que determina pautas de acción y que establece exigencias y desafíos que modelan el mundo interno $($ selff). Es necesario advertir sobre la unilateralidad del movimiento, pues es un asunto de relevancia para la praxis educativa contemporánea: la sociedad influye al sujeto, pero son pocas las posibilidades de que éste impacte en el orden de lo social, pues está atomizado y meramente abocado a ajustarse a la productividad escolar y/o a las dinámicas de un mundo complejo.

En línea con ello, el ideario del equilibrio juega un papel articulador de los lenguajes psicológicos emergentes en estos contextos de política educativa. La recurrente apelación al equilibrio y la armonía sitúa en el sujeto la responsabilidad de generar las disposiciones psicológicas y psicosociales necesarias para afrontar un mundo social desafiante y repleto de riesgos y adversidades. A su vez, se potencia la idea de los jóvenes como únicos responsables de su salud, en tanto el bienestar, el equilibrio socioemocional, los hábitos de vida saludable, son cuestiones que se constituyen desde la performance del sujeto, pero sin considerar condicionantes estructurales, como aquellas de tipo históricas, raciales, de clase social, de género, entre otras, que operan y se entrelazan al construir esta idea del ser integral-ser saludable. En ambos casos, el mundo social y educativo son relatados con una tonalidad aséptica y estéril, evidenciando que la sociedad es un mero y pulcro escenario de desenvolvimiento y que el sujeto, evitando todo tipo de desviación y disrupción, debe orientarse funcionalmente hacia ella.

En este contexto, el lenguaje psicológico cumple, ideológicamente, una función no sólo normalizadora y justificadora de las intervenciones que parecen ser necesarias para la población, sino que, ante todo, se posiciona cómplice ante las racionalidades y di- námicas que cotidianamente operan al servicio de la opresión de gran parte de los seres humanos que habitan el continente latinoamericano. En las palabras siempre vigentes de Freire(2012), estas prácticas reafirman el sentido bancario de la educación latinoamericana, considerando que,

en verdad, lo que pretenden los opresores es transformar la mentalidad de los oprimidos y no la situación que los oprime. A fin de lograr una mejor adaptación a la situación que, a la vez, permita una mejor forma de dominación. Para esto, utilizan una concepción "bancaria" de la educación a la que vinculan todo el desarrollo de una acción social de carácter paternalista, en que los oprimidos reciben el simpático nombre de "asistidos". Son casos individuales, meros "marginalizados", que discrepan de la fisonomía general de la sociedad. Esta es buena, organizada y justa. Los oprimidos son la patología de las sociedades sanas, que precisan por esto mismo ajustarlos a ellas, transformando sus mentalidades de hombres "ineptos y perezosos (p.64).

Sutilmente, desde su complicidad ideológica, los lenguajes psicológicos incorporados en educación pretenden contribuir a equilibrar y armonizar el mundo interno de sujetos latinoamericanos, sin ningún cuestionamiento de las condiciones que generan dinámicas de opresión. $\mathrm{Al}$ respecto, consideramos que es una necesidad histórica y un desafío para la teorización e investigación educativa, revisar profundamente qué es lo que hacemos con el lenguaje psicológico y qué es lo que hace éste con nosotros, en nuestras complejas configuraciones como sujetos individuales y colectivos, y en los dinamismos que constituyen nuestras vidas y trayectorias educativas. Pero, por sobre todas las cosas, pensamos que es necesario posicionarse desde una vigilancia epistemológica hacia la Psicología en su relación con la educación, revisando cuidadosamente sus modos del decir y del hacer, particularmente en lo referente al carácter dualista de su estructuración discursiva y a su participación cómplice en las dinámicas que buscan moldear y ajustar servilmente a diversos sujetos a los requerimientos de las élites nacionales y supranacionales. 


\section{Revista Caribeña de Investigación Educativa | 2020, 4(1), 62-80}

\section{Referencias}

Ball, S. (2006). What is policy? Texts, trajectories and toolboxes. Discourse: Studies in the Cultural Politics of Education, 13(2), 10-17.

https://doi.org/10.1080/0159630930130203

Ball, S. J. (2009). Privatising education, privatising education policy, privatising educational research: Network governance and the 'competition state'. Journal of Education Policy, 24(1), 83-99. https://doi.org/10.1080/02680930802419474

Ball, S. (2013). Foucault, Power and Education. Routledge. https://doi.org/10.4324/9780203078662

Ball, S. (2016). Neoliberal education? Confronting the slouching beast. Policy Futures in Education, 14 (8), 1046-1059.

https://doi.org/10.1177/1478210316664259

Bekerman, Z., \& Zembylas, M. (2018). Psychologized language in education. Denaturalizing a Regime of Truth. Palgrave Macmillan.

Brown, K. (2017). The governance of vulnerability: regulation, support and social divisions in action. International Journal of Sociology and Social Policy, 37(11-12), 667-682.

https://doi.org/10.1108/IJSSP-04-2016-0049

Brown, K., Ecclestone, K., \& Emmel, N. (2017). The many faces of vulnerability. Social Policy and Society, 16(3), 497-510.

https://doi.org/10.1017/S1474746416000610

Brunila, K. (2011). The Projectisation, Marketisation and Therapisation of Education. European Educational Research Journal, 10(3), 421-432. https://doi.org/10.2304/eerj.2011.10.3.421

De Vos, J. (2013). Psychologization and the Subject of Late Modernity. Palgrave Macmillan.

De Vos, J. (2015). Deneurologizing Education? From Psychologisation to Neurologisation and Back. Studies in Philosophy and Education, 34(3), 279-295. https://doi.org/10.1007/s11217-014-9440-5

De Vos, J. (2016). The metamorphoses of the brain:
Neurologisation and its discontents. Palgrave Macmillan. https://doi.org/10.1057/978-1-137-50557-6

Ecclestone, K., \& Brunila, K. (2015). Governing emotionally vulnerable subjects and "therapisation" of social justice. Pedagogy, Culture \& Society, 23(4), 485-506.

https://doi.org/10.1080/14681366.2015.1015152

Ecclestone, K. (2016). Behaviour change policy agendas for "vulnerable" subjectivities: the dangers of therapeutic governance and its new entrepreneurs. Journal of Education Policy, 32(1), 48-62.

https://doi.org/10.1080/02680939.2016.1219768

Ecclestone, K., \& Rawdin, C. (2016). Reinforcing the "disminished" subject? The implications of the "vulnerability zeitgeist" for well-being in educational settings. Cambridge Journal of Education, 46(3), 377393.

https://doi.org/10.1080/0305764X.2015.1120707

Ecclestone, K. (2017). Therapeutic governance of psyco-emotionally vulnerable citizens: new subjectivities, new experts and new dangers. In J. Pykket, R. Jones \& M. Whitehead (Eds.). Psychological governance and public policy. Governing the mind, brain and behavior (pp.1-20). Routledge.

https://doi.org/10.1080/02680939.2016.1219768

Fairclough, N. (2003). Analysing discourse. Textual analysis for social research. Routledge.

Fairclough, I., \& Fairclough, N. (2012). Political Discourse Analysis. A method for advanced students. Routledge.

Fairclough, N. (2018). CDA as dialectical reasoning. In J. Flowerdew \& J. Richardson (Eds.). The Routledge handbook of critical discourse studies (pp.18-38). Routledge. https://doi.org/10.4324/9781315739342-2

Falabella, A. (2019). The ethics of competition: accountability policy enactment in Chilean schools' everyday life. Journal of Education Policy, 34(6), 1-23. https://doi.org/10.1080/02680939.2019.1635272

Flowerdew, J., \& Richardson, J. (2018). The Routledge handbook of critical discourse studies. Routledge. 
https://doi.org/10.4324/9781315739342

Foucault, M. (1992). El orden del discurso. Tusquets.

Foucault, M. (2006). Seguridad, territorio y población. Fondo de Cultura Económica.

Foucault, M. (2008). Vigilar y castigar. Nacimiento de la prisión. Siglo XXI.

Freire, P. (2012). Pedagogía del oprimido. Siglo XXI.

Fuller, K., \& Stevenson, H. (2019). Global education reform: understanding the movement. Educational Review, 71(1), 1-4.

https://doi.org/10.1080/00131911.2019.1532718

Gentili, P. (2011). Pedagogía de la igualdad. Ensayos contra la educación excluyente. Siglo XXI.

Harvey, D. (2015). Breve historia del neoliberalismo. Akal.

Lewis, S. (2016). Governing schooling through "what Works": the OECD's PISA for Schools. Journal of Education Policy, 32(3), 1-22.

https://doi.org/10.1080/02680939.2016.1252855

Maroy C., Pons X., \& Dupuy C. (2019) Trajectories. In C. Maroy, X. Pons (Eds.). Accountability Policies in Education (pp.115-150). Educational Governance Research.

https://doi.org/10.1007/978-3-030-01285-4_5

Miller, P., \& Rose, N. (2008). Governing the present. Polity Press.

Ministerio de Educación de Chile (2014). Otros Indicadores de Calidad Educativa. MINEDUC. https:// bit.ly/2QBgiAY

Ministerio de Educación de Chile (2015). Manual Bienestar en la Escuela. Buen Clima Escolar. MINEDUC. https://bit.ly/2N65aK4

Ministerio de Educación de Ecuador (2016). Modelo de Funcionamiento de los Departamentos de Consejería Estudiantil. Ministerio de Educación del Ecuador. https://bit.ly/2t2qsBw

Moretti, R., \& Energici, M. (2019). Perspectiva gu- bernamental e historia de la psicología. Medición de la inteligencia y la aptitud en la educación chilena (1925-1967). En E. Hevia, F. Reiter \& G. Salas (Eds.). Historias de la psicología. Contribuciones y reconstrucciones parciales (pp.255-285). Universidad Alberto Hurtado. https://bit.ly/36FeV9S

Palacios, D. (2018). Trabajando en la emergencia. Los procesos de recontextualización de las politicas educativas desde el discurso de psicólogos/as que trabajan en escuelas emergentes en el marco de la Ley de Subvención Escolar Preferencial (Tesis Doctoral). Universidad de Chile, Santiago de Chile.

Palacios, D., Hidalgo, F., Cornejo, R., \& Suárez, N. (2019). Análisis Político de Discurso: Herramientas conceptuales y analíticas para el estudio crítico de políticas educativas en tiempos de reforma global. Archivos Analiticos de Politicas Educativas, 27(47), 1-31. https://doi.org/10.14507/epaa.27.4269

Parra, D. (2015). Un análisis historiográfico sobre las relaciones entre psicología y educación en Chile (1889-1973). Revista de Historia de la Psicología, 36(2), 94-115. https://bit.ly/2FvvYiR

Pykett, J., Jones, R. \& Whitehead, M. (2017). Psychological Governance and Public Policy. Routledge. https://doi.org/10.4324/9781315680248

Rautalin, M., Alasuutari, P., \& Vento, E. (2018). Globalisation of education policies: does PISA have an effect?. Journal of Education Policy, 34(4), 1-23. https://doi.org/10.1080/02680939.2018.1462890

Rose, N. (1996). Inventing ourselves: Psychology, Power and Personhood. Cambridge University Press. https://doi.org/10.1017/cbo9780511752179

Rose, N. (1999). Governing the soul. The shaping of the private self. Free Association Books.

Rose, N. (2006). The politics of life itself: Biomedicine, power \& subjectivity in the twenty-first century. Princeton University Press.

Salhberg, P. (2016). The Global Educational Reform Movement and Its Impact on_Schooling. In K. Mundy, A. Green, B. Lindgard, \& A. Verger (Eds.). 
The Handbook of Global Educational Policy (pp.128148). Routledge.

https://doi.org/10.1002/9781118468005

Verger, A., Moschetti, M., \& Fontdevila, C. (2017). La privatización educativa en América Latina: Una cartografia de politicas, tendencias y trayectorias. Education International. https://bit.ly/3ajNJzP

Verger, A., Parcerisa, L., \& Fontdevila, C. (2019). The growth and spread of large-scale assesments and test-based accountabilities: A political sociology of global education reforms. Educational Review, 71, 5-30.

https://doi.org/10.1080/00131911.2019.1522045

Verger, A., Zancajo, A., \& Fontdveila, C. (2016). La economía política de la privatización educativa: Políticas, tendencias y trayectorias desde una perspectiva comparada. Revista Colombiana de Educación, 70, 47-78.

https://doi.org/10.17227/01203916.70rce47.78

\section{CÓMO CITAR:}

Palacios-Díaz, D., Hidalgo-Kawada, F., \& Saavedra-Stuardo, P. (2020).

Psicologización y lenguaje en educación: Analizando discursivamente políticas educativas latinoamericanas contemporáneas. Revista Caribeña de Investigación

Educativa (RECIE), 4(1), 62-88. https://doi.org/10.32541/recie.2020.v4i1.pp62-88 